Please do not remove this page

RMIT

UNIVERSITY

\title{
Crystallization of hard-sphere colloids: deviations from classical nucleation theory
}

Bryant, Gary; Schoepe, Hans-Jaochim; Van Megen, William

https://researchrepository.rmit.edu.au/esploro/outputs/9921859752701341/filesAndLinks?institution=61RMIT_INST\&index=null

Bryant, G., Schoepe, H.-J., \& Van Megen, W. (2006). Crystallization of hard-sphere colloids: deviations from classical nucleation theory. Proceedings of the 2006 International Conference on Nanosciences and Nanotechnology, 123-126. https://doi.org/10.1109/ICONN.2006.340566

Published Version: https://doi.org/10.1109/ICONN.2006.340566

Repository homepage: https://researchrepository.rmit.edu.au

(C) 2006 IEEE

Downloaded On 2023/04/26 15:14:49 +1000

Please do not remove this page 


\title{
Crystallization of hard-sphere colloids - deviations from classical nucleation theory
}

\author{
Gary Bryant*, Hans Joachim Schöpe ${ }^{\dagger}$ and William van Megen*. \\ *Applied Physics, School of Applied Sciences, RMIT University \\ GPO Box 2476V, Melbourne 2001 \\ Email: gary.bryant@rmit.edu.au \\ 'Institut für Physik, Johannes Gutenberg-Universität Mainz, \\ WA KOMET 336, Staudingerweg 7, D-55128 Mainz, Germany \\ Email: jschoepe@uni-mainz.de
}

\begin{abstract}
The creation of three-dimensional ordered colloidal crystals, for application in a range of nanotechnologies, has been a goal for many researchers in the past few years. The main difficulty in creating macroscopic sized crystals of densely packed colloidal particles is that colloidal particles always have a range of particle sizes - ie, they are polydisperse. In this paper we study the crystallization kinetics of a hard-sphere colloid with a well defined Gaussian polydispersity. We find that crystallization occurs in two stages, and does not follow the simple classical nucleation picture. We discuss the implications of these results for research into colloidal crystals as possible nano-materials.
\end{abstract}

Keywords- colloids; crystallization; polydispersity

\section{INTRODUCTION}

There has been a great deal of interest over the past decade in the creation of three-dimensional ordered colloidal crystals. Such crystals, with lattice constants comparable to the wavelength of visible light, have potential applications as photonic crystals [1], optical filters and switches [2], and chemical sensors [3]. Several methods have been developed to control the self-assembly of the colloidal crystals, including colloidal epitaxy [4] and the use of temperature gradients [5].

Although there has been significant progress in making ordered colloidal crystals in the last few years, the goal of macroscopic, defect free colloidal crystals is yet to be achieved. Such crystals are usually compromised by poly-crystallinity, along with point defects and dislocations.

The grain size in these polycrystalline materials is determined by the interplay between nucleation and growth, even under conditions where crystallization is driven by external fields. In order to produce high quality, macroscopic, defect free crystals, we need a full understanding of the mechanisms underlying crystal nucleation and growth.

The major difference between crystallization in atomic systems and in colloidal suspensions, apart from their vastly different time scales, is that any colloidal preparation contains a range of particle sizes around a mean value. The consequences of this inherent polydispersity on nucleation and crystal growth has been largely ignored by all but a few workers. Recently however, the effect of polydispersity has been the focus of both theory and simulation [6-8] and experimental studies [9-11]. These studies have demonstrated that even for small polydispersities $(<10 \%)$, there is local fractionation or segregation of particle sizes [8], which has a strong retarding effect on nucleation [9-10]. These effects are magnified when the particles have a skewed particle size distribution [9], or when two components with slightly different sizes are mixed [11].

In this paper we present the crystallization kinetics of a colloidal sample with a well defined Gaussian polydispersity. We find that crystallization occurs in two stages, and does not follow the simple classical nucleation picture. We discuss the implications of these results for the growth of large scale colloidal crystals. Some of the results presented here have been reported elsewhere [12].

\section{METHODS}

The experimental methods and analysis used here are described elsewhere [10-12] and are summarized here briefly. The particles consist of a copolymer core of methylmethacrylate and tri-fluoroethylacrylate, with a stabilising coating of poly-12-hydroxystearic acid, about $10 \mathrm{~nm}$ thick, bonded to the surface. The particle size distribution is shown in fig. 1, as determined using scanning electron microscopy (SEM).

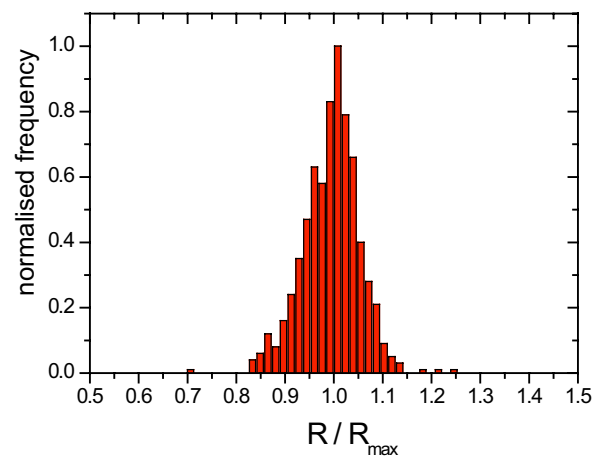

Figure 1. Particle size distribution for the particles used here, determined by scanning electron microscopy. The results are from an analysis of approximately 1000 particles. The distribution is fit well by a Gaussian with an average particle radius of $\mathrm{R}_{\mathrm{EM}}=315 \mathrm{~nm}$, and a polydispersity of $4.8 \%$. 
Approximately 1000 particles were analysed, yielding a Gaussian distribution with an average particle radius of $\mathrm{R}_{\mathrm{EM}}=315 \mathrm{~nm}$, and a polydispersity of $4.8 \%$. These results are consistent with independent measurements using both Static Light Scattering and Dynamic Light Scattering. The latter yielded a hydrodynamic radius of $\mathrm{R}_{\mathrm{H}}=320 \mathrm{~nm}$. Details of the characterization of the particle size distribution will be published elsewhere [13]. The particles are refractive index matched to the suspending solvent cis-decalin $(n=1.483$ at $25^{\circ} \mathrm{C}$ ), and the radii reported include any swelling effects.

The particles behave as near perfect hard spheres with an interaction range of $\sim 12 \mathrm{~nm}$ [14], with freezing at a volume fraction of $\phi=0.505$ and melting at $\phi=0.538$. Prior to each measurement the samples were tumbled for $>24$ hours to shear melt any crystals, and then left undisturbed. The crystals which form are studied using laser light Bragg scattering, analogous to X-ray powder diffraction. Measurements are made using a purpose built Bragg spectrometer, which measures the structure factor of the crystallites $S_{x t a l}$ as a function of the scattering vector q. As crystallization proceeds, the following parameters can be extracted from the measurements:

First, by integrating the crystalline structure factor $\mathrm{S}_{\mathrm{xtal}}$ over the area of the main Bragg reflection we can extract the crystallinity $X$, the fraction of the sample which is crystalline:

$$
X(t)=c \cdot \int S_{x t a l}(q, t) d q
$$

where c is a scaling constant. Second, the position of the peak maximum $\mathrm{q}_{\max }$ gives the lattice spacing of the crystal, and hence for a given value of particle radius $\mathrm{R}$, the crystalline volume fraction $\phi_{x t a l}$ may be determined:

$$
\phi_{x t a l}(t)=\frac{2\left[q_{\max }(t) R\right]^{3}}{9 \sqrt{3} \pi^{2}}
$$

The third parameter which may be directly determined from the Bragg peak is the average linear dimension (or average size) of the crystals, $<\mathrm{L}(\mathrm{t})>$, which is determined from the full width at half maximum of the peak $\delta \mathrm{q}$ :

$$
<L(t)>=\frac{2 \pi K}{\delta q(t)}
$$

where $\mathrm{K}$ is the Scherrer constant $(\mathrm{K}=1.107$ for a spherical shaped crystal).

Given the crystallinity and the average linear dimension, we can determine the total number of crystals $\mathrm{N}_{\text {xtal }}$ in the scattering volume $\mathrm{V}_{\text {scatt }}$ :

$$
N_{x t a l}(t)=\frac{X(t) \cdot V_{s c a t t}}{\left\langle L^{3}(t)\right\rangle}=\frac{X(t) \cdot V_{s c a t t}}{\alpha \cdot\langle L(t)\rangle^{3}}
$$

where $\alpha$ relates the average volume to the cube of the average length, and is of order 1.25.

Finally, we can determine the nucleation rate density $\mathrm{J}$, which is defined as the rate at which crystals appear in the available liquid (free) volume:

$$
J(t)=\frac{1}{(1-X(t))} \frac{d}{d t} \frac{X(t)}{\left\langle L^{3}(t)\right\rangle}
$$

\section{RESULTS}

Fig. 2 shows typical examples of the crystalline structure factor peak (main interlayer reflection) from crystallites forming from the melt as a function of time. Fig. 2a shows the first hour following the quench, where only one broad peak is observed growing slowly in intensity. Fig. $2 \mathrm{~b}$ shows the period between 1 and 2 hours following the quench, when a second peak begins to appear. The primary peak corresponds to the face centred cubic (fcc) (111) peak (or equivalently, the hexagonal close packed (hcp) (002) peak). The secondary peak corresponds to hcp (100). The hcp (101) peak becomes clearly visible at later times. The crystallites have now converted from a precursor structure (Fig. 2a) into the random hexagonal close packed (rhcp) structure (Fig. 2b). This structure (a mixture of $\mathrm{ABC}$ and $\mathrm{ABA}$ layers) is typical for hard sphere colloidal crystals. Following the conversion, the intensity increases rapidly (Fig. 2c), but the structure remains essentially unchanged. We define these three stages of growth, based on the number of crystals, as: (a) induction; (b) conversion; and (c) final nucleation.

From these structure factors, the time dependent behaviour of the crystallinity and related parameters are determined, as shown in fig. 3. The panels represent: the crystallinity (fig. 3a); the crystalline volume fraction (fig. 3b); the average crystal size (fig. 3c); the number of crystallites in the scattering volume (fig. 3d); and the nucleation rate density (fig. 3e). The time intervals corresponding to the three stages of growth (induction, conversion and final nucleation) are indicated by the horizontal markers in fig. $3 \mathrm{c}$.

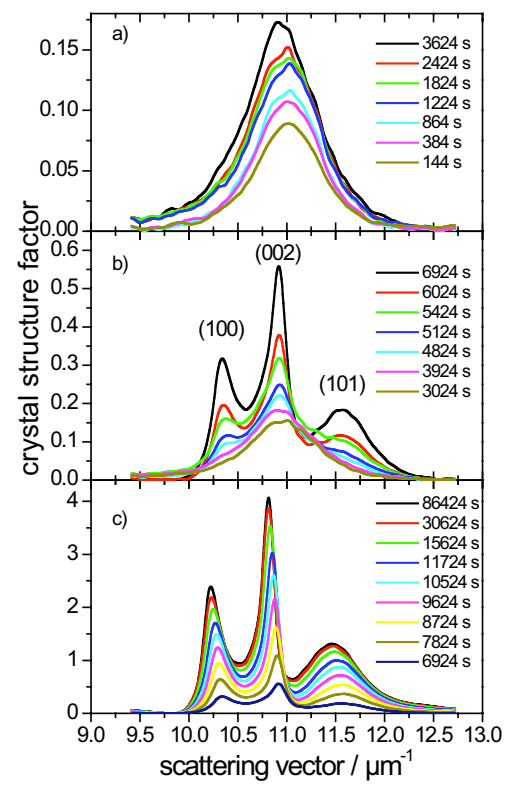

Figure 2. Crystalline structure factors at a volume fraction near melting $(\Phi=$ 0.538 ) during the three time intervals (a) induction, (b) conversion and (c) final nucleation. Note the different scales. 


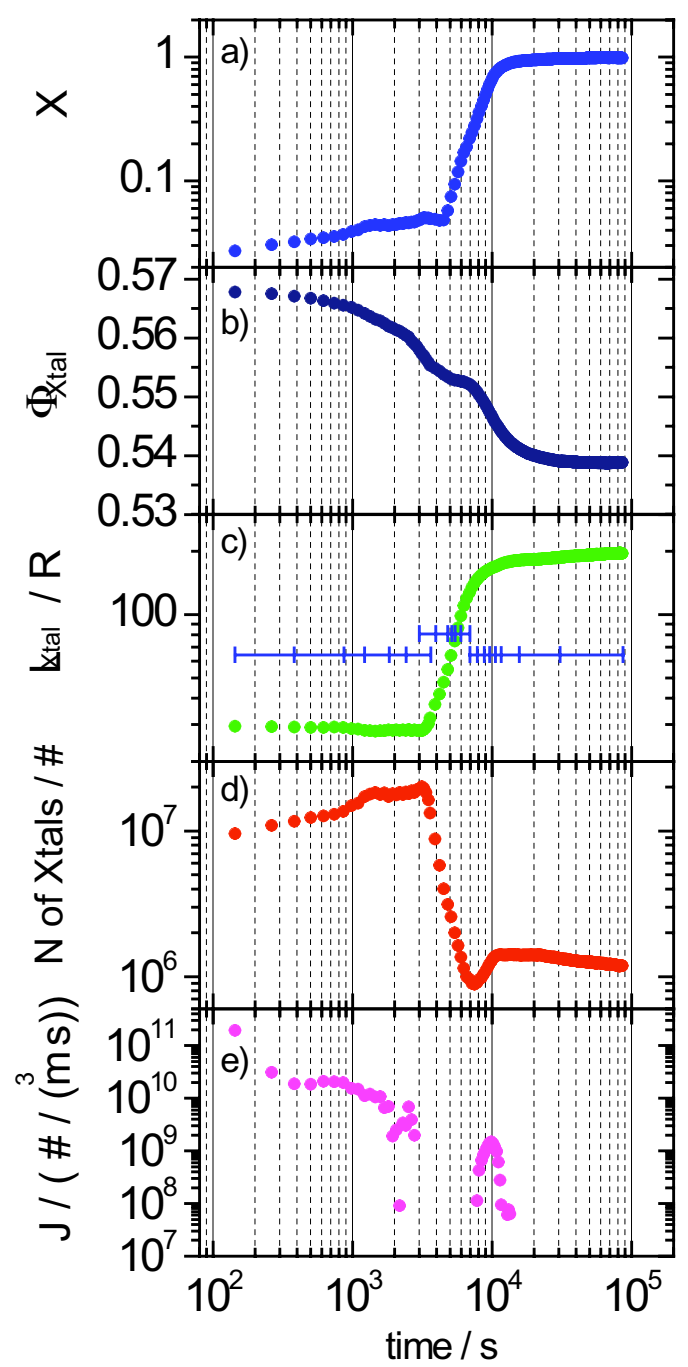

Figure 3. Extracted parameters from the data shown in Fig. 2: a) crystallinity; b) crystalline volume fraction; c) average linear dimension; d) absolute number of crystals in the scattering volume; e) nucleation rate densities

\section{DISCUSSION}

We will discuss each of the stages in turn.

Induction phase - a Bragg peak is discernable from the fluid background within about 200 seconds following the quench. During the induction stage, the amount of crystal in the samples (3a) slowly rises, while the average crystal size remains relatively constant $(3 \mathrm{c})$, suggesting that these precursor crystallites are unstable and do not grow. Consequently, the total number of crystallites ( $3 \mathrm{~d}$ ) increases during this period. The crystallites that form are compact, starting with a volume fraction of 0.565 , and slowly dropping as the crystals expand (3b). During this period, the nucleation rate density starts high (when there is no crystal), and slowly drops as more and more crystallites form and there is less available fluid volume (3e).
The behaviour observed during this initial phase is consistent with the formation of small, compressed crystallites. If these crystallites were close packed (hcp or fcc) structures, one would expect that in this highly undercooled (or more correctly, overpacked) system the crystals would rapidly grow throughout the sample. The fact that they do not, suggests that the crystallites do not have fcc or hop packings. From the data presented here we cannot predict which packings might be present. However, molecular dynamics simulations [16] and confocal microscopy $[17,18]$ both indicate that at early times a range of other packings can occur (eg icosahedral, simple cubic or body centred cubic). Such packings would be consistent with the observations reported here.

Conversion phase - in this second stage, the crystallinity continues to grow slowly (3a), but this is now accompanied by a rapid reduction in the number of crystallites (3d) and a sharp increase in the average crystal size (3c). The nucleation rate density is here undefined, as the number of crystals is falling. Clearly during this period, the crystallites are growing, as the average crystal size approaches its maximum value. However, only a small fraction of the sample $(\sim 10 \%)$ is crystalline at the end of this stage of growth.

We interpret the observed behaviour as being due to the precursor crystallites slowly converting into rhcp crystallites, and then beginning to grow. The drop in the number of crystals implies that either the growing crystallites are absorbing unconverted crystallites during their growth, or that the precursor crystallites disappear as the growing crystallites consume more of the sample volume.

Final nucleation - the final phase consists of an accelerated nucleation process where both the number of crystallites $(3 \mathrm{~d})$ and the total amount of crystal (3a) grows rapidly, but the average crystal size $(3 \mathrm{c})$ rises more slowly. The nucleation rate density (3e) rises sharply in this period.

During this stage the crystallinity grows by a factor of 8 , with very little increase in the crystal size. Clearly in this region, the formation of new crystallites dominates over growth - why should this be the case? One might expect that the large crystals would swiftly grow throughout the available volume.

The explanation lies in the polydispersity [10-12]. Consider a growing crystal consisting of particles of a certain size $R_{c}$. At the crystal-fluid interface, further growth will proceed by the addition of more particles to the crystal interface - however, if there are several particles of different radii within a short range of the interface, then on average, a particle with radius $R_{c}$, will have a higher probability of joining the crystal. If a smaller or larger particle joins the crystal, it will produce a less than efficient packing, and the crystal will have less chance of continuing to grow.

In this way, growing crystals will selectively filter the surrounding fluid for particles closer to the average radius. As the crystal continues to grow, the concentration of non-optimal particles around the crystal will increase, and eventually, this will slow further growth. Eventually, the concentration of these particles will become sufficiently high that growth can only occur via incorporation of the particles - at this point further 
growth becomes less energetically favourable than the formation and growth of new crystallites elsewhere in the sample. This process sets up the conditions for the final nucleation event to occur at long times.

Following the final nucleation stage, the sample is essentially fully crystalline, and the crystallinity, crystalline volume fraction, average crystal size and number of crystals plateau to long term values. Any slow changes occurring at these long times are due to ripening effects. Eventually, on time scales longer than those shown here, the effect of gravity becomes important.

One of the important results to emerge from this analysis is that the nucleation rate density - rather than being a constant, as predicted by classical nucleation theory - has two distinct regions: a region at early times, corresponding to the formation of the precursor crystallites, and a region at later times corresponding to the main nucleation process. These results show that for real (polydisperse) colloidal systems, the nucleation rate density varies markedly through the crystallization process, contrary to the naive view of classical nucleation theory.

In addition to being of fundamental importance, these results have consequences for attempts to create large colloidal crystals for applications in nanotechnology. Such methods as templating and the use of external fields will also be limited by the same polydispersity effects observed here. As crystals grow, the same fractionation effects will occur, and the inclusion of particles of non-optimal size will induce defects in the crystal structure.

One approach to relieve this problem might be to try to develop particles with significantly lower polydispersities however, this is very difficult. Most methods or particle preparation produce polydispersities in the range of $2 \%$ and up, which is large enough to ensure these effects occur. In any case, smaller polydispersities may be counter productive - if the particles are all identical in size, nucleation will proceed so quickly that a polycrystalline structure is inevitable.

\section{CONCLUSIONS}

In this abstract we have demonstrated that the traditional view of crystallization does not apply to real colloidal systems, where polydispersity ensures that nucleation processes are considerably more complicated. For the pursuit of methods for producing controlled, macroscopic colloidal crystals, polydispersity is both an asset and a challenge: on one hand the presence of polydispersity slows crystal growth sufficiently that it may be controlled; on the other hand, polydispersity limits the maximum size colloidal crystals can have without containing defects. Polydispersity should therefore be an important consideration in any attempts to develop large scale colloidal crystals.

\section{ACKNOWLEDGMENT}

HJS would like to thank the Alexander von Humbold Foundation for providing the Feodor-Lynen Fellowship.

\section{REFERENCES}

[1] Joannopoulos, J. D., P. R. Villeneuve, S. and Fan, "Photonic crystals: putting a new twist on light," Nature 386, 143-149, 1997.

[2] Pan, G., R. Kesavamoorthy, and S. A. Asher, "Optically nonlinear Bragg diffracting nanosecond optical switches," Phys. Rev. Lett. 78, 38603863, 1997.

[3] Holtz, J. H. and S. A. Asher, "Polymerized colloidal crystal hydrogel films as intelligent chemical sensing materials," Nature 389, 829-832, 1997.

[4] van Blaaderen, A., R. Ruel, and P. Wiltzius, "Template-directed colloidal crystallization," Nature 385, 321-324, 1997.

[5] Cheng, Z., W. B. Tussel, and P. M. Chaikin, "Controlled growth of hardsphere colloidal crystals," Nature, 401, 893-895, 1999.

[6] Auer, S., and D. Frenkel, "Supression of crystal nucleation in polydisperse colloids due to increase of the surface energy," Nature (London) 413, 711-713, 2001

[7] Bartlett, P., J. "Freezing in polydisperse colloidal suspensions," Phys. Cond. Mat. 12, A275-A280, 2000.

[8] Kofke, D. A. and P. G. Bolhuis, "Freezing of polydisperse hard spheres," Phys. Rev. E 59, 618-622, 1999.

[9] Martin, S., G. Bryant, and W. van Megen, "Observation of a smecticlike crystalline structure in polydisperse colloids," Phys. Rev. Lett., 90, 255701, 2003.

[10] Martin, S., G. Bryant, and W. van Megen, "Crystallization kinetics of polydisperse colloidal hard spheres: Evidence for local fractionation," Phys. Rev. E, 67, 061405, 2003.

[11] Martin, S., G. Bryant, and W. van Megen, "Crystallization kinetics of polydisperse colloidal hard spheres. II. Binary mixtures," Phys. Rev. E, 71, 021404, 2005

[12] Schöpe, H.J., G. Bryant and W. van Megen, "Two Step Crystallization Kinetics in Colloidal Hard-Sphere Systems." Phys. Rev. Lett. 96: $175701,2006$.

[13] Schöpe, H.J., G. Bryant and W. van Megen, (to be published).

[14] Bryant, G., S. R. Williams, L. Qian, I. K. Snook, E. Perez and F. Pincet. "How hard is a colloidal "hard-sphere" interaction?" Phys. Rev. E, 66, 060501(R), 2002.

[15] Francis, P., S. Martin, G. Bryant, W. van Megen and P. A. Wilksch, "A Bragg scattering spectrometer for studying crystallization of colloidal suspensions," Rev. Sci. Instrum. 73: 3878-3884, 2002.

[16] O'Malley, B. and I. K. Snook, "Structure of hard-sphere fluid and precursor structures to crystallization," J. Chem. Phys. 123: 054511, 2005

[17] Gasser, U., A. Schofield and D. A. Weitz, "Local order in a supercooled colloidal fluid observed by confocal microscopy," J. Phys. Condens. Matter 15, S375-S380, 2003.

[18] Gasser, U., E. R. Weeks, A. Schofield, P. N. Puset and D. A. Weitz, "Real-space imaging of nucleation and growth in colloidal crystallization," Science 292: 258-262, 2001. 